\title{
SOME BIANCHI TYPE I VISCOUS FLUID COSMOLOGICAL MODELS WITH A VARIABLE COSMOLOGICAL CONSTANT
}

\author{
ANIRUDH PRADHAN* and PURNIMA PANDEY \\ Department of Mathematics, Hindu Post-graduate College, Zamania-232 331, \\ Ghazipur, U. P., India; \\ E-mail:acpradhan@yahoo.com,pradhan@iucaa.ernet.in
}

\begin{abstract}
Some Bianchi type I viscous fluid cosmological models with a variable cosmological constant are investigated in which the expansion is considered only in two direction i.e. one of the Hubble parameter $\left(H_{1}=\frac{A_{4}}{A}\right)$ is zero. The viscosity coefficient of bulk viscous fluid is assumed to be a power function of mass density whereas the coefficient of shear viscosity is considered as constant in first case whereas in other case it is taken as proportional to scale of expansion in the model. The cosmological constant $\Lambda$ is found to be positive and is a decreasing function of time which is supported by results from recent supernovae Ia observations. Some physical and geometric properties of the models are also discussed.
\end{abstract}

Keywords: Cosmology; Bianchi type I universe; viscous fluid models; variable cosmological constant.

\section{Introduction}

Models with a dynamic cosmological term $\Lambda(t)$ are becoming popular as they solve the cosmological constant problem in a natural way. There are significant observational evidence for the detection of Einstein's cosmological constant, $\Lambda$ or a component of material content of the universe that varies slowly with time and space to act like $\Lambda$. Recent cosmological observations by High -Z Supernova Team and Supernova Cosmological Project (Garnavich et al., 1998; Perlmutter et al., 1997, 1998, 1999; Riess et al., 1998; Schmidt et al., 1998) suggest the existence of a positive cosmological constant $\Lambda$ with magnitude $\Lambda\left(G \hbar / c^{3}\right) \approx 10^{-123}$. These observations on magnitude and red-shift of type Ia supernova suggest that our universe may be an accelerating function of the cosmological density in the form of the cosmological $\Lambda$ term. Earlier researches on this topic are contained in Zeldovich (1968), Weinberg (1972), Dolgov (1983, 1990), Bertolami (1986), Ratra and Peebles (1988), Carroll, Press and Turner (1992). Some of the recent discussions on the cosmological constant "problem" and consequences on cosmology with a time-varying cosmological constant have been

* Corresponding Author

(c) 2018 Kluwer Academic Publishers. Printed in the Netherlands. 
discussed by Dolgov (1993,1997), Tsagas and Maartens (2000), Sahni and Starobinsky (2000), Peebles (2003), Padmanabhan (2003), Vishwakarma (1999, 2000, 2001, 2002), and Pradhan et al. (2001, 2002, $2003,2004)$. This motivates us to study the cosmological models in which $\Lambda$ varies with time.

The distribution of matter can be satisfactorily described by a perfect fluid due to the large scale distribution of galaxies in our universe. However, observed physical phenomena such as the large entropy per baryon and the remarkable degree of isotropy of the cosmic microwave background radiation, suggest analysis of dissipative effects in cosmology. Furthermore, there are several processes which are expected to give rise to viscous effects. These are the decoupling of neutrinos during the radiation era and the decoupling of radiation and matter during the recombination era. Bulk viscosity is associated with the GUT phase transition and string creation. Misner $(1967,1968)$ has studied the effect of viscosity on the evolution of cosmological models. The role of viscosity in cosmology has been investigated by Weinberg (1971). Nightingale (1973), Heller and Klimek (1975) have obtained a viscous universes without initial singularity. The model studied by Murphy (1973) possessed an interesting feature in which big bang type of singularity of infinite space-time curvature does not occur to be a finite past. However, the relationship assumed by Murphy between the viscosity coefficient and the matter density is not acceptable at large density. Roy and Prakash (1977) have investigated a plane symmetric cosmological models representing a viscous fluid with free gravitational field of non-degenerate Petrov type I in which coefficient of shear viscosity is constant. Bali and Jain $(1987,1988)$ have obtained some expanding and shearing viscous fluid cosmological models in which coefficient of shear viscosity is proportional to rate of expansion in the model and the free gravitational field is Petrov type $\mathrm{D}$ and non-degenerate. The effect of bulk viscosity on the cosmological evolution has been investigated by a number of authors in the framework of general theory of relativity (Padmanabhan and Chitre, 1987; Johri and Sudarshan, 1988; Maartens, 1995; Zimdahl, 1996; Pradhan et al., 1997; Kalyani and Singh, 1997; Singh et al., 1998; Pradhan et al., 2001, 2002). This motivates to study cosmological bulk viscous fluid model.

Recently Bali and Jain (1997) have investigated Bianchi type I viscous fluid cosmological models. Motivated by the situations discussed above, in this paper, we shall focus on the problem with varying cosmological constant in presence of bulk and shear viscous fluid in an expanding universe. We do this by extending the work of Bali and Jain 
(1997) by including varying cosmological constant and the coefficient of bulk viscosity as function of time. This paper is organized as follows: The metric and the field equations are presented in section 2 . In section 3 , we deal with the solution of the field equations in presence of viscous fluid. In section 3.1, we consider the coefficient of shear viscosity $(\eta)$ as constant whereas in section 3.2, $\eta$ is taken as proportional to scale of expansion in the model. Section 4 includes the solution in absence of shear viscosity. In section 5 , we have given the concluding remarks.

\section{The Metric and Field Equations}

We consider the Bianchi type I metric in the form

$$
d s^{2}=-d t^{2}+d x^{2}+B^{2} d y^{2}+C^{2} d z^{2},
$$

where $B$ and $C$ are functions of $t$ alone. This metric depicts the case

when one of the Hubble parameters (here $H_{1}=\frac{A_{4}}{A}$ ) is zero, i. e. the expansion is only in two directions. The kinematic parameters are then related as $\theta=-3 \sigma_{1}^{1}$. This condition leads to the metric (1).

The Einstein's field equations (in gravitational units $c=1, G=1$ ) read as

$$
R_{i}^{j}-\frac{1}{2} R g_{i}^{j}+\Lambda g_{i}^{j}=-8 \pi T_{i}^{j},
$$

where $R_{i}^{j}$ is the Ricci tensor; $R=g^{i j} R_{i j}$ is the Ricci scalar; and $T_{i}^{j}$ is the stress energy-tensor in the presence of bulk stress given by Landau and Lifshitz (1963)

$$
\begin{aligned}
T_{i}^{j}=(\rho+p) v_{i} v^{j} & +p g_{i}^{j}-\eta\left(v_{i ;}^{j}+v_{; i}^{j}+v^{j} v^{l} v_{i ; l}+v_{i} v^{l} v_{; l}^{j}\right) \\
& -\left(\xi-\frac{2}{3} \eta\right) \theta\left(g^{j}{ }_{i}+v_{i} v^{j}\right) .
\end{aligned}
$$

Here $\rho, p, \eta$ and $\xi$ are the energy density, isotropic pressure, coefficient of shear viscosity and bulk viscous coefficient respectively and $v_{i}$ is the flow vector satisfying the relations

$$
g_{i j} v^{i} v^{j}=-1 .
$$

The semicolon $(;)$ indicates covariant differentiation. We choose the coordinates to be comoving, so that

$$
v^{1}=0=v^{2}=v^{3}, v^{4}=1 .
$$


The Einstein's field equations (2) for the line element (1) has been set up as

$$
\begin{gathered}
-8 \pi\left[p-\left(\xi-\frac{2}{3} \eta\right) \theta\right]=\frac{B_{44}}{B}+\frac{C_{44}}{C}+\frac{B_{4} C_{4}}{B C}+\Lambda, \\
-8 \pi\left[p-2 \eta \frac{B_{4}}{B}-\left(\xi-\frac{2}{3} \eta\right) \theta\right]=\frac{C_{44}}{C}+\Lambda, \\
-8 \pi\left[p-2 \eta \frac{C_{4}}{C}-\left(\xi-\frac{2}{3} \eta\right) \theta\right]=\frac{B_{44}}{B}+\Lambda, \\
8 \pi \rho=\frac{B_{4} C_{4}}{B C}+\Lambda,
\end{gathered}
$$

where the suffix 4 at the symbols $A$ and $B$ denotes ordinary differentiation with respect to $t$ and $\theta$ is the scalar of expansion given by

$$
\theta=v_{; i}^{i}
$$

\section{Solution of the Field Equations}

We have revisited the solutions obtained by Bali and Jain (1997). Equations (6) - (9) are four independent equations in seven unknowns $A$, $B, \rho, p, \eta, \xi$ and $\Lambda$. For the complete determinacy of the system, we need three extra conditions. The research on exact solutions is based on some physically reasonable restrictions used to simplify the Einstein equations.

From Eqs. (6) - (8), we obtain

$$
\frac{B_{44}}{B}+\frac{B_{4} C_{4}}{B C}=-16 \pi \eta \frac{B_{4}}{B}
$$

and

$$
\frac{B_{44}}{B}-\frac{C_{44}}{C}=-16 \pi \eta\left(\frac{B_{4}}{B}-\frac{C_{4}}{C}\right) .
$$

Equation (12) on integration leads to

$$
C^{2}\left(\frac{B}{C}\right)_{4}=L e^{-16 \pi \eta t}
$$

where $L$ is a constant of integration. Setting $B C=\mu$ and $\frac{B}{C}=\nu$ in Eqs. (11), (12) and (13) lead to

$$
\mu_{44}+16 \pi \eta \mu_{4}=0
$$

and

$$
\frac{\nu_{4}}{\nu}=\frac{L}{\mu} e^{-16 \pi \eta t}
$$


Equation (14) on integration leads to

$$
\mu_{4}=M e^{-16 \pi \eta t}
$$

where $M$ is an integrating constant.

Here we consider two cases:

\subsection{CASE I : LET $\eta=\mathrm{CONSTANT}=a(\mathrm{SAY})$}

In this case Eq. (16) on integration gives

$$
\mu=N-\frac{M}{16 \pi a} e^{-16 \pi a t},
$$

where $N$ is a constant of integration.

Equation (15) on integration leads to

$$
\nu=\alpha\left(16 \pi a N-M e^{-16 \pi a t}\right)^{\frac{L}{M}},
$$

where $\alpha$ is an integrating constant.

Now we set

$$
\begin{aligned}
e^{-16 \pi a t} & =\cos 2 \sqrt{16 \pi a} \tau, \\
\alpha & =\frac{1}{(16 \pi a)^{\frac{L}{M}}}
\end{aligned}
$$

and

$$
N=\frac{M}{16 \pi a} .
$$

Using the above Eqs. (19) - (21) in (17) and (18), we obtain

$$
\mu=2 M\left(\frac{\sin \sqrt{16 \pi a} \tau}{\sqrt{16 \pi a}}\right)^{2}
$$

and

$$
\nu=(2 M)^{\frac{L}{M}}\left(\frac{\sin \sqrt{16 \pi a} \tau}{\sqrt{16 \pi a}}\right)^{\frac{2 L}{M}} .
$$

From above Eqs. (22) and (23), we obtain

$$
\begin{aligned}
& B^{2}=\mu \nu=(2 M)^{1+\frac{L}{M}}\left(\frac{\sin \sqrt{16 \pi a} \tau}{\sqrt{16 \pi a}}\right)^{2+\frac{2 L}{M}} \\
& C^{2}=\frac{\mu}{\nu}=(2 M)^{1-\frac{L}{M}}\left(\frac{\sin \sqrt{16 \pi a} \tau}{\sqrt{16 \pi a}}\right)^{2-\frac{2 L}{M}}
\end{aligned}
$$


Hence the metric (1) reduces to the form

$$
\begin{gathered}
d s^{2}=-4\left(\frac{\tan 2 \sqrt{16 \pi a} \tau}{\sqrt{16 \pi a}}\right) d \tau^{2}+d x^{2}+(2 M)^{1+\frac{L}{M}}\left(\frac{\sin \sqrt{16 \pi a} \tau}{\sqrt{16 \pi a}}\right)^{2+\frac{2 L}{M}} d y^{2} \\
+(2 M)^{1-\frac{L}{M}}\left(\frac{\sin \sqrt{16 \pi a} \tau}{\sqrt{16 \pi a}}\right)^{2-\frac{2 L}{M}} d z^{2}
\end{gathered}
$$

After suitable transformation of coordinates metric (26) reduces to the form

$d s^{2}=-4\left(\frac{\tan 2 k T}{k}\right) d T^{2}+d X^{2}+\left(\frac{\sin k T}{k}\right)^{2+\frac{2 L}{M}} d Y^{2}+\left(\frac{\sin k T}{k}\right)^{2-\frac{2 L}{M}} d z^{2}$

where $k=\sqrt{16 \pi a}$.

The pressure and density for the model (27) are given by

$$
\begin{gathered}
8 \pi p=\frac{1}{4 M^{2}}\left(\frac{k}{\sin k T}\right)^{4}\left[M(M-L) \sin ^{2} k T \cos 2 k T-\frac{(L+M)}{4} \cos 2 k T \times\right. \\
\left.\{(L+M) \cos 2 k T-2 M\}+16 \pi M^{2}\left(\xi-\frac{2}{3} a\right) \cos 2 k T\left(\frac{\sin k T}{k}\right)^{2}\right]-\Lambda \\
8 \pi \rho=\frac{1}{4 M^{2}}\left(\frac{k}{\sin k T}\right)^{4}\left[\frac{\left(M^{2}-L^{2}\right)}{4} \cos ^{2} 2 k T\right]+\Lambda
\end{gathered}
$$

For the specification of $\xi$, we assume that the fluid obeys an equation of state of the form

$$
p=\gamma \rho
$$

where $\gamma(0 \leq \gamma \leq 1)$ is constant. Thus, given $\xi(t)$ we can solve for the cosmological parameters. In most of the investigation involving bulk viscosity is assumed to be a simple power function of the energy density (Pavon, 1991; Maartens, 1995; Zimdahl, 1996)

$$
\xi(t)=\xi_{0} \rho^{n},
$$

where $\xi_{0}$ and $n$ are constants. If $n=1$, Equation (23) may correspond to a radiative fluid (Weinberg, 1972). However, more realistic models (Santos, 1985) are based on $n$ lying in the regime $0 \leq n \leq \frac{1}{2}$.

On using (31) in (28), we obtain

$8 \pi p=\frac{1}{4 M^{2}}\left(\frac{k}{\sin k T}\right)^{4}\left[M(M-L) \sin ^{2} k T \cos 2 k T-\frac{(L+M)}{4} \cos 2 k T \times\right.$ 
$\left.\{(L+M) \cos 2 k T-2 M\}+16 \pi M^{2}\left(\xi_{0} \rho^{n}-\frac{2}{3} a\right) \cos 2 k T\left(\frac{\sin k T}{k}\right)^{2}\right]-\Lambda$

3.1.1. Model I: Solution for $\xi=\xi_{0}$

When $n=0$, Equation (31) reduces to $\xi=\xi_{0}=$ constant. Hence in this case Equation (32), with the use of (29) and (30), leads to

$$
\begin{aligned}
64 \pi M^{2}(1+\gamma) \rho= & \left(\frac{k}{\sin k T}\right)^{4} \cos 2 k T\left[M^{2}-L^{2}+2\left(L^{2}+M^{2}\right) \sin ^{2} k T+\right. \\
& \left.32 \pi M^{2}\left(\xi_{0}-\frac{2 a}{3}\right)\left(\frac{\sin k T}{k}\right)^{2}\right]
\end{aligned}
$$

Eliminating $\rho(t)$ between Eqs. (29) and (33), we have

$$
\begin{gathered}
2(1+\gamma) \Lambda=\cos 2 k T\left[(1-\gamma)\left(M^{2}-L^{2}\right)+\right. \\
\left.2\left\{\left(3 M^{2}+L^{2}\right)+\left(M^{2}-L^{2}\right) \gamma\right\} \sin ^{2} k T+64 \pi M^{2}\left(\xi_{0}-\frac{2 a}{3}\right)\left(\frac{\sin k T}{k}\right)^{2}\right]
\end{gathered}
$$

3.1.2. Model I: Solution for $\xi=\xi_{0} \rho$

When $n=1$, Equation (31) reduces to $\xi=\xi_{0} \rho$. Hence in this case Equation (32), with the use of (29) and (30), leads to

$$
\begin{gathered}
16 \pi \rho=\frac{k^{4} \cos 2 k T}{M^{2}\left[2(1+\gamma) \sin ^{2} k T-k^{2} \xi_{0} \cos 2 k T\right] \sin ^{2} k T} \times \\
{\left[\frac{M^{2}-L^{2}}{2}+\left(L^{2}+M^{2}\right) \sin ^{2} k T-\frac{8 \pi a}{3} \cos 2 k T\left(\frac{k}{\sin k T}\right)^{2}\right]}
\end{gathered}
$$

Eliminating $\rho(t)$ between (29) and (35), we have

$$
\begin{gathered}
\Lambda=-\frac{\left(M^{2}-L^{2}\right) \cos ^{2} 2 k T}{16 M^{2}}\left(\frac{k}{\sin k T}\right)^{4}+ \\
\frac{k^{4} \cos 2 k T}{2 M^{2}\left[2(1+\gamma) \sin ^{2} k T-k^{2} \xi_{0} \cos 2 k T\right] \sin ^{2} k T} \times \\
{\left[\frac{M^{2}-L^{2}}{2}+\left(L^{2}+M^{2}\right) \sin ^{2} k T-\frac{8 \pi a}{3} \cos 2 k T\left(\frac{k}{\sin k T}\right)^{2}\right]}
\end{gathered}
$$

Our study for these models shows constant value of cosmological constant $(\Lambda)$ for large values of time and do not decrease with time (this 
means that the universe is not expanding or may be steady state condition). In this case, detailed study shows that the scalar of expansion $\theta$ does not increase with time. Our study is inconsistent with work done by Bali and Jain (1997). Bali and Jain claim that the universe is expanding which does not match with our result. Also the claim of minimum and maximum expansion rate in $\theta$ is reflection of periodicity of trigonometrical functions involved there. We are trying to find feasible interpretation and situations relevant to this case. Further study is in progress.

Some Physical Aspects of the Models:

With regard to the kinematic properties of the velocity vector $v^{i}$ in the metric (27), a straight forward calculation leads to the following expressions for the scalar of expansion $(\theta)$ and for the shear $(\sigma)$ of the fluid.

$$
\begin{gathered}
\theta=\frac{1}{2} \cos 2 k T\left(\frac{k}{\sin k T}\right)^{2} \\
\sigma^{2}=\frac{1}{48}\left(1+\frac{3 L^{2}}{M^{2}}\right) \cos ^{2} 2 k T\left(\frac{k}{\sin k T}\right)^{4}
\end{gathered}
$$

The rotation $\omega$ is identically zero. The non-vanishing components of conformal curvature tensor are

$$
\begin{gathered}
C_{12}^{12}=\frac{\cos 2 k T}{48 M^{2}}\left(\frac{k}{\sin k T}\right)^{4}\left[M^{2}-L^{2}-2 L(3 M-L) \sin ^{2} k T\right] \\
C_{13}^{13}=\frac{\cos 2 k T}{48 M^{2}}\left(\frac{k}{\sin k T}\right)^{4}\left[M^{2}-L^{2}+2 L(3 M+L) \sin ^{2} k T\right] \\
C_{14}^{14}=\frac{\cos 2 k T}{24 M^{2}}\left(\frac{k}{\sin k T}\right)^{4}\left[L^{2}-M^{2}-2 L^{2} \sin ^{2} k T\right]
\end{gathered}
$$

The models represent shearing, non-rotating and Petrov type I nondegenerate in general, in which the flow is geodetic. However, if $L=0$ then spacetime reduces to Petrov type $D$. The model starts expanding at $T>0$ but the initial expansion is slow. When $T$ is closer to $\pi / 2 k$, it has stiff rise in the expansion and then decreases. This shows the case of oscillation. It is observed that the expansion is minimum at $T=0$ or $T=\pi / k$. The large values of $\theta$ near $T=\pi / 2 k$ is reflection of trigonometric property. But expansion remains finite. The expression for $\frac{\sigma}{\theta}$ and $\frac{\rho}{\theta^{2}}$ are found to be

$$
\frac{\sigma}{\theta}=\frac{1}{2 \sqrt{3}}\left(1+\frac{3 L^{2}}{M^{2}}\right)^{\frac{1}{2}}
$$




$$
\frac{\rho}{\theta^{2}}=\frac{1}{32 \pi M^{2} \cos ^{2} 2 k T}\left[\left(M^{2}-L^{2}\right) \cos ^{2} 2 k T+16 M^{2} \Lambda\left(\frac{\sin k T}{k}\right)^{4}\right]
$$

From Equation (42), it can be seen that shear is proportional to scalar of expansion $\theta$ in the model. Since $\lim _{T \rightarrow \infty} \frac{\sigma}{\theta} \neq 0$, hence the models do not approach isotropy for large values of $T$.

The rate of expansion $H_{i}$ (Hubble parameters) in the direction of $X$, $Y, Z$ are given by

$$
\begin{gathered}
H_{1}=0 \\
H_{2}=\frac{(M+L)}{4 M T^{2}} \\
H_{2}=\frac{(M-L)}{4 M T^{2}}
\end{gathered}
$$

\subsection{CASE II: LET $\eta=\beta \theta$, WHERE $\beta$ IS AN ARBITRARY CONSTANT.}

In this case Equation (14) reduces to

$$
\mu \mu_{44}+16 \pi \beta \mu_{4}^{2}=0,
$$

which on integration leads to

$$
\mu=\left[(1+16 \pi \beta)\left(k_{1} t+k_{2}\right)\right]^{\frac{1}{(1+16 \pi \beta)}},
$$

where $k_{1}$ and $k_{2}$ are constants of integration.

Equation (12) reduces to

$$
\mu\left(\frac{\nu_{4}}{\nu}\right)_{4}=-(1+16 \pi \beta) \frac{\mu_{4}}{\mu},
$$

which on integration leads to

$$
\nu=k_{3}\left(k_{1} t+k_{2}\right)^{\frac{k_{4}}{k_{1}(1+16 \pi \beta)}}
$$

where $k_{3}$ and $k_{4}$ are constants of integration.

From Equations (48) and (50), we obtain

$$
\begin{gathered}
B^{2}=\mu \nu=k_{3} k_{5}\left(k_{1} t+k_{2}\right)^{k_{6}\left(k_{1}+k_{4}\right),} \\
C^{2}=\frac{\mu}{\nu}=\frac{k_{5}}{k_{3}}\left(k_{1} t+k_{2}\right)^{k_{6}\left(k_{1}-k_{4}\right)},
\end{gathered}
$$

where

$$
k_{5}=(1+16 \pi \beta)^{\frac{1}{(1+16 \pi \beta)}},
$$




$$
k_{6}=\frac{1}{k_{1}(1+16 \pi \beta)} .
$$

Hence the metric (1) reduces to

$$
d s^{2}=-d t^{2}+d x^{2}+k_{3} k_{5}\left(k_{1} t+k_{2}\right)^{k_{6}\left(k_{1}+k_{4}\right)} d y^{2}+\frac{k_{5}}{k_{3}}\left(k_{1} t+k_{2}\right)^{k_{6}\left(k_{1}-k_{4}\right)} d z^{2}
$$

After suitable transformation of coordinates, the metric (55) takes the form

$$
d s^{2}=-\frac{1}{k_{1}^{2}} d T^{2}+d X^{2}+T^{k_{6}\left(k_{1}+k_{4}\right)} d Y^{2}+T^{k_{6}\left(k_{1}-k_{4}\right)} d Z^{2}
$$

The pressure and density for the model (56) are given by

$$
\begin{gathered}
8 \pi p=-\frac{k_{1}^{2} k_{6}\left(k_{1}+k_{4}\right)}{4 T^{2}}\left(k_{1} k_{6}+k_{4} k_{6}-2\right) \\
+\frac{8 \pi k_{1}^{2} k_{6}}{T}\left[\frac{\beta k_{1} k_{6}}{3 T}\left(k_{1}-k_{4}\right)+\xi\right]-\frac{k_{2}}{2 k_{5}^{2} T^{2 k_{1} k_{6}}}-\Lambda \\
8 \pi \rho=\frac{k_{1}^{2} k_{6}^{2}\left(k_{1}^{2}-k_{4}^{2}\right)}{4 T^{2}}+\Lambda
\end{gathered}
$$

On using (31) in (57), we obtain

$$
\begin{gathered}
8 \pi p=-\frac{k_{1}^{2} k_{6}\left(k_{1}+k_{4}\right)}{4 T^{2}}\left(k_{1} k_{6}+k_{4} k_{6}-2\right) \\
+\frac{8 \pi k_{1}^{2} k_{6}}{T}\left[\frac{\beta k_{1} k_{6}}{3 T}\left(k_{1}-k_{4}\right)+\xi_{0} \rho^{n}\right]-\frac{k_{2}}{2 k_{5}^{2} T^{2 k_{1} k_{6}}}-\Lambda
\end{gathered}
$$

3.2.1. Model I: Solution for $\xi=\xi_{0}$

When $n=0$, Equation (59), with the use of (58) and (30), leads to

$$
\begin{gathered}
8 \pi(1+\gamma) \rho=\frac{k_{1}^{2} k_{6}\left(k_{1}+k_{4}\right)\left(1-k_{4} k_{6}\right)}{2 T^{2}} \\
+\frac{8 \pi k_{1}^{2} k_{6}}{T}\left[\frac{\beta k_{1} k_{6}}{3 T}\left(k_{1}-k_{4}\right)+\xi_{0}\right]-\frac{k_{2}}{2 k_{5}^{2} T^{2 k_{1} k_{6}}}
\end{gathered}
$$

Eliminating $\rho(t)$ between Equations (58) and (60), we have

$$
\begin{gathered}
(1+\gamma) \Lambda=\frac{k_{1}^{3} k_{6}\left(k_{1}+k_{4}\right)}{T}\left[2-\left\{k_{1}+k_{4}+\left(k_{1}-k_{4}\right) \gamma\right\} k_{6}\right] \\
+\frac{8 \pi k_{1}^{2} k_{6}}{T}\left[\frac{\beta k_{1} k_{6}}{3 T}\left(k_{1}-k_{4}\right)+\xi_{0}\right]-\frac{k_{2}}{2 k_{5}^{2} T^{2 k_{1} k_{6}}}
\end{gathered}
$$


3.2.2. Model I: Solution for $\xi=\xi_{0} \rho$

When $n=1$, Equation (59), with the use of (58) and (30), leads to

$$
\begin{gathered}
8 \pi\left[1+\gamma-\frac{k_{1}^{2} k_{6} \xi_{0}}{T}\right] \rho=\frac{k_{1}^{2} k_{6}^{2}}{6 T^{2}}\left[3\left(k_{1}+k_{4}\right)\left(1-k_{4} k_{6}\right)\right. \\
\left.+16 \pi \beta k_{1}\left(k_{1}-3 k_{4}\right)\right]-\frac{k_{2}}{2 k_{5}^{2} T^{2 k_{1} k_{6}}}
\end{gathered}
$$

Eliminating $\rho(t)$ between (58) and (62), we have

$$
\begin{gathered}
{\left[1+\gamma-\frac{k_{1}^{2} k_{6} \xi_{0}}{T}\right] \Lambda=\frac{k_{1}^{2} k_{6}\left(k_{1}+k_{4}\right)}{4 T^{2}}\left[2-\left(k_{1}+k_{4}\right) k_{6}\right.} \\
\left.-\left(k_{1}-k_{4}\right) k_{6}\left\{\gamma-\frac{k_{1}^{2} k_{6} \xi_{0}}{T}\right\}\right]+\frac{8 \pi \beta k_{1}^{3} k_{6}^{2}\left(k_{1}-3 k_{4}\right)}{3 T^{2}}-\frac{k_{2}}{2 k_{5}^{2} T^{2 k_{1} k_{6}}}
\end{gathered}
$$

From Equations (61) and (63), we observe that the cosmological constant in both models is a decreasing function of time and it approaches a small value as time progresses (i. e. the present epoch), which explains the small value of $\Lambda$ at present.

\section{Some Physical Aspects of the Models:}

With regard to the kinematic properties of the velocity vector $v^{i}$ in the metric (56), a straight forward calculation leads to the following expressions for the scalar of expansion $(\theta)$ and for the shear $(\sigma)$ of the fluid.

$$
\begin{gathered}
\theta=\frac{k_{1}^{2} k_{6}}{T} \\
\sigma^{2}=\frac{k_{1}^{2} k_{6}^{2}\left(k_{1}^{2}+k_{4}^{2}\right)}{2 T^{2}}
\end{gathered}
$$

The rotation $\omega$ is identically zero. The non-vanishing components of conformal curvature tensor are

$$
\begin{gathered}
C_{12}^{12}=\frac{k_{1}^{2} k_{6}}{12 T^{2}}\left[k_{1}-3 k_{4}+k_{4} k_{6}\left(3 k_{1}-k_{4}\right)\right] \\
C_{13}^{13}=\frac{k_{1}^{2} k_{6}}{12 T^{2}}\left[k_{1}+3 k_{4}-k_{4} k_{6}\left(3 k_{1}+k_{4}\right)\right] \\
C_{14}^{14}=\frac{k_{1}^{2} k_{6}}{6 T^{2}}\left(k_{4}^{2} k_{6}-k_{1}\right)
\end{gathered}
$$

The models represent an expanding, shearing but non-rotating universe in general. The models explode with a big bang at $T=0$ and the expansion in the models stops at $T=\infty$. When $k_{1}=0$ then $\theta=0$, which implies that $\eta=0$. Therefore, viscosity is due to expansion in the model. We take $k_{1} \neq 0$. The spacetime is Petrov type I non-degenerate. 
However, if $k_{4}=0$, the spacetime reduces to Petrov type ID. For large values of $T$, the spacetime is conformally flat. The expressions $\frac{\sigma}{\theta}$ and $\frac{\rho}{\theta^{2}}$ are found to be

$$
\begin{gathered}
\frac{\sigma}{\theta}=\frac{\left(k_{1}^{2}+3 k_{4}^{2}\right)^{\frac{1}{2}}}{2 \sqrt{3} k_{1}} \\
\frac{\rho}{\theta^{2}}=\frac{1}{32 \pi k_{1}^{4} k_{6}^{2}}\left[k_{1}^{2} k_{6}^{2}\left(k_{1}^{2}-k_{6}^{2}\right)+4 \Lambda T^{2}\right]
\end{gathered}
$$

The rate of expansion $H_{i}$ (Hubble parameters) in the direction of $X$, $Y, Z$ are given by

$$
\begin{gathered}
H_{1}=0 \\
H_{2}=\frac{k_{1} k_{6}\left(k_{1}+k_{4}\right)}{2 T} \\
H_{3}=\frac{k_{1} k_{6}\left(k_{1}-k_{4}\right)}{2 T}
\end{gathered}
$$

Since $\lim _{T \rightarrow \infty} \frac{\sigma}{\theta} \neq 0$, hence the models do not approach isotropy for large values of $T$.

\section{Solution in Absence of Shear Viscosity}

When $\eta \rightarrow 0$, then the metric (27) leads to

$$
d s^{2}=-16 T^{2} d T^{2}+d X^{2}+T^{\left(2+\frac{2 L}{M}\right)} d Y^{2}+T^{\left(2-\frac{2 L}{M}\right)} d Z^{2}
$$

The pressure and density for the model (74) are given by

$$
\begin{gathered}
8 \pi p=\frac{1}{16 M^{2} T^{4}}\left[64 \pi M^{2} \xi T^{2}+M^{2}-L^{2}\right]-\Lambda \\
8 \pi \rho=\frac{\left(M^{2}-L^{2}\right)}{16 M^{2} T^{4}}+\Lambda
\end{gathered}
$$

\subsection{MODEL I: SOLUTION FOR $\xi=\xi_{0}$}

When $n=0$, Equation (31) reduces to $\xi=\xi_{0}$ (constant). Hence in this case Equation (75), with the use of (29) and (76), leads to

$$
8 \pi(1+\gamma) \rho=\frac{1}{8 M^{2} T^{4}}\left[32 \pi M^{2} \xi_{0} T^{2}+M^{2}-L^{2}\right]
$$

Eliminating $\rho(t)$ between (76) and (77), we obtain

$$
(1+\gamma) \Lambda=\frac{1}{16 M^{2} T^{4}}\left[64 \pi M^{2} \xi_{0} T^{2}-\left(M^{2}-L^{2}\right)(1-\gamma)\right]
$$




\subsection{MODEL II: SOLUTION FOR $\xi=\xi_{0} \rho$}

When $n=1$, Equation (31) reduces to $\xi=\xi_{0} \rho$. Hence in this case Equation (75), with the use of (29) and (76), leads to

$$
\left[2(1+\gamma) T^{2}-\xi_{0}\right] \rho=\frac{M^{2}-L^{2}}{32 \pi M^{2} T^{2}}
$$

Eliminating $\rho(t)$ between (76) and (79), we have

$$
\left[2(1+\gamma) T^{2}-\xi_{0}\right] \Lambda=\frac{\left(M^{2}-L^{2}\right)}{16 M^{2} T^{4}}\left[4 T^{2}-2(1+\gamma) T^{2}+\xi_{0}\right]
$$

From Equations (78) and (80), we observe that the cosmological constant in both the models is a decreasing function of time and it approaches a small positive value for large time (i. e. the present epoch), when $M^{2}-L^{2}>0$, which is supported by the results from recent type Ia supernovae observations (Garnavich et al., 1998; Perlmutter et al.,1997, 1998, 1999; Riess et al., 1998; Schmidt et al., 1998).

Some Physical Aspects of the Models:

The scalar of expansion $(\theta)$, shear $(\sigma)$ and the non-vanishing components of conformal curvature tensor are given by

$$
\begin{gathered}
\theta=\frac{1}{2 T^{2}} \\
\sigma^{2}=\frac{\left(M^{2}+3 L^{2}\right)}{48 M^{2} T^{4}} \\
C_{12}^{12}=C_{13}^{13}=\frac{\left(M^{2}-L^{2}\right)}{48 M^{2} T^{4}} \\
C_{14}^{14}=\frac{\left(L^{2}-M^{2}\right)}{24 M^{2} T^{4}}
\end{gathered}
$$

The expressions $\frac{\sigma}{\theta}$ and $\frac{\rho}{\theta^{2}}$ are as follows:

$$
\begin{gathered}
\frac{\sigma}{\theta}=\frac{1}{2 \sqrt{3} M}\left(M^{2}+3 L^{2}\right)^{\frac{1}{2}} \\
\frac{\rho}{\theta^{2}}=\frac{1}{32 \pi M^{2}}\left[\left(M^{2}-L^{2}\right)+M^{2} T^{4}\right]
\end{gathered}
$$

From Equation (85), it is observed that the shear is proportional to the expansion $\theta$ in the models. The models explode with a big bang at $T=0$ and the expansion in the models stops at $T=\infty$. The spacetime is non-degenerate Petrov type $\mathrm{I}$ in absence of viscosity and if $L=0$, then spacetime is Petrov type $\mathrm{D}$. For large values of $T$, the spacetime is conformally flat. Since $\lim _{T \rightarrow \infty} \frac{\sigma}{\theta} \neq 0$, hence the models 
do not approach isotropy for large values of $T$. The metric (74) has singularity at $T=0$. The models start from $T=0$ with big bang and goes on expanding till $T=\infty$.

\section{Conclusions}

Some Bianchi type I anisotropic cosmological models with a viscous fluid as the source of matter are obtained. Generally, the models are expanding, shearing and non-rotating. It is observed that in the presence of viscosity, the spacetime is Petrov type I D, whereas without viscosity the spacetime is conformally flat. It is seen that the solutions obtained by Bali and Jain (1997) are particular cases of our solutions. In all these models, we observe that they do not approach isotropy for large values of time $T$. These models are new and different from those models obtained by Bali and Jain $(1987,1988)$ in which free gravitational field was assumed to be Petrov type $\mathrm{D}$ and non-degenerate for Marder (1958).

The cosmological constant in all models given in sections 3.2 and 4 are decreasing function of time and they all approach a small positive value as time increases (i.e., the present epoch). The values of cosmological "constant" for these models are found to be small and positive which are supported by the results from recent supernovae Ia observations recently obtained by the High - Z Supernova Team and Supernova Cosmological Project ( Garnavich et al., 1998 ; Perlmutter et al., 1997, 1998, 1999; Riess et al., 1998; Schmidt et al. , 1998). In section 3.1, our study is inconsistent with work done by Bali and Jain (1997). Our study for these models shows constant value of cosmological constant for large values of time and do not decrease with time i. e. universe is not expanding or may be steady state condition. Bali and Jain claim that the universe is expanding which does not match with our results. We are trying to find feasible interpretation to this case. Further study is in progress.

\section{Acknowledgements}

One of the authors (A. Pradhan) thanks to the Inter-University Cen-

tre for Astronomy and Astrophysics, India for providing facility under Associateship Programmes where part of work was carried out. 


\section{References}

Bali R. and Jain, V.C.: 1987, Astrophys. Space Sci., 139, 175.

Bali R. and Jain, V.C.: 1988, Astrophys. Space Sci., 141, 207.

Bali R. and Jain, V.C.: 1997, Astrophys. Space Sci., 254, 13.

Bertolami, O.: 1986, Nuovo Cimento, B 93, 36.

Bertolami, O.: 1986, Fortschr. Phys. 34, 829.

Carroll, S.M., Press, W.H. and Turner, E.L.: 1992, Ann. Rev. Astron. Astrophys. 30, 499.

Chakrabarty, I., Pradhan, A. and Saste, N.N.: 2001, Int. J. Mod. Phys. D 10, 741.

Dolgov, A.D.: 1983, in The Very Early Universe, eds. Gibbons, G.W., Hawking, S.W. and Siklos, S.T.C. (Cambridge University Press).

Dolgov, A.D., Sazhin, M.V. and Zeldovich, Ya.B.: 1990, Basic Modern Cosmology, (Editions Frontiers).

Dolgov, A.D. and Silk, J.: 1993, Phys. Rev. D 47, 3144.

Dolgov, A.D.: 1993, Phys. Rev. D 48, 2499.

Dolgov, A.D.: 1997, Phys. Rev. D 55, 5881.

Garnavich, P.M. et al.: 1998a, Astrophys. J. 493, L53, astro-ph/9710123; 1998b, Astrophys. J. 509, 74, astro-ph/9806396.

Heller, M. and Klimek, Z.: 1975, Astrophys. Space Sci. 33, 37.

Johri, V.B. and Sudarshan, R.: 1988, Phys. Lett. A 132, 316.

Kalyani, D. and Singh, G.P.: 1997, in New Direction in Relativity and Cosmology, eds. V. de Sabbata and T. Singh, Hadronic Press, U S A, p.41.

Marder, L.: 1958, Proc. Roy. Soc. A 246, 139.

Maartens, R.: 1995, Class Quantum Gravit. 12, 1455.

Misner, C.W.: 1967, Nature 214, 40.

Misner, C.W.: 1968, Astrophys. J. 151, 431.

Murphy, G.L.: 1973, Phys. Rev. D 8, 4231.

Nightingale, J.P.: 1973, Astrophys. J. 185, 105.

Padmanabhan, T.: 2003, Phys. Rep. 380, 235; hep-th/0212290.

Padmanabhan, T. and Chitre, S.M.: 1987, Phys. Lett. A 120, 433.

Pavon, D., Bafaluy, J. and Jou, D.: 1991, Class. Quant. Grav. 8, 357; 1996, "Proc. Hanno Rund Conf. on Relativity and Thermodynamics", Ed. S. D. Maharaj, University of Natal, Durban, p. 21.

Perlmutter, S. et al.: 1997, Astrophys. J. 483, 565, astro-ph/9608192; 1998, Nature 391, 51, astro-ph/9712212; 1999, Astrophys. J. 517, 565, astro-ph/9608192.

Peebles, P.J.E. and Ratra, B.: 2003, Rev. Mod. Phys. 75, 559, astro-ph/0207347.

Pradhan, A., Sarayakar, R.V. and Beesham, A.: 1997, Astr. Lett. Commun. 35, 283.

Pradhan, A., Yadav, V.K. and Chakrabarty, I.: 2001, Int. J. Mod. Phys. D 10, 339. Pradhan, A., V.K. Yadav and Saste, N.N.: 2002, Int. J. Mod. Phys. D 11, 857.

Pradhan, A. and Yadav, V.K.: 2002, Int. J. Mod. Phys. D 11, 839.

Pradhan, A. and Aotemshi, I.: 2002, Int. J. Mod. Phys. D 11, 1419.

Pradhan, A. and Pandey, H.R.: 2003, Int. J. Mod. Phys. D 12, 941.

Pradhan, A. and Pandey, O.P.: 2003, Int. J. Mod. Phys. D 12, 1299.

Pradhan, A. and Singh, S.K.: 2004, Int. J. Mod. Phys. D 13, 503.

Pradhan, A. and Srivastav, S.K. and Jotania, K.R.: 2004,Czech. J. Phys. 54, 255.

Pradhan, A. and Yadav, L. and Yadav, A.K.: 2004,Czech. J. Phys. 54, 487.

Ratra, B. and Peebles, P.J.E.: 1988, Phys. Rev. D 37, 3406.

Riess, A.G. et al.: 1998, Astron. J. 116, 1009, astro-ph/9805201.

Roy, S.R. and Prakash, S.: 1977, Ind. J. pure Appl. Math. 8, 723. 
Sahni, V. and Starobinsky, A.: 2000, Int. J. Mod. Phys. D 9, 373, gr-qc/9904398.

Santos, N.O., Dias, R.S. and Banerjee, A.: 1985, J. Math. Phys. 26, 878.

Schmidt, B. P. et al.: 1998, Astrophys. J. 507, 46, astro-ph/9805200.

Singh, T., Beesham, A. and Mbokazi, W. S.: 1998, Gen. Rel. Grav. 30, 537.

Tsagas, C.G. and Maartens, R.: 2000, Class. Quant. Grav. 17, 2215, gr-qc/9912044.

Vishwakarma, R. G. and Abdussattar: 1999, Phys. Rev. D 60, 063507.

Vishwakarma, R.G.: 2000, Class. Quant. Grav. 17, 3833.

Vishwakarma, R.G.: 2001, Class. Quant. Grav. 18, 1159.

Vishwakarma, R.G.: 2001, Gen. Rel. Grav. 33, 1973.

Vishwakarma, R.G.: 2002, Mon. Not. R. Astron. Soc. 331, 776.

Vishwakarma, R.G.: 2002, Class. Quant. Grav. 19, 4747.

Weinberg, S.: 1971, Astrophys. J. 168, 175.

Weinberg, S.: 1972, Gravitation and Cosmology, Wiley, New York.

Zeldovich, Ya. B.: 1968, Sov. Phys.-Uspekhi 11, 381.

Zimdahl, W.: 1996, Phys. Rev. D 53, 5483. 